\title{
Comparison of Apical Axial Derotation between Adolescent Idiopathic and Neuromuscular Scoliosis with Pedicle Screw Instrumentation
}

\author{
Hitesh N Modi, Seung-Woo Suh, S Srinivasalu, Satyen Mehta, Jae-Hyuk Yang \\ Scoliosis Research Institute, Department of Orthopedics, Korea University Guro Hospital, Seoul, Korea
}

Study Design: A retrospective study.

Purpose: To compare outcomes of apical derotation with pedicle screws in idiopathic and neuromuscular scoliosis (NMS).

Overview of Literature: No information about apical derotation in NMS with pedicle screws is available.

Methods: We performed deformity correcting surgery using pedicle screw constructs on 12 adolescent idiopathic scoliosis (AIS) patients (mean age 14.1 years) and 16 NMS patients (mean age 16.5 years). Preoperative, postoperative, and final follow-up radiographs were analyzed for Cobb's angle and pelvic obliquity, while apical rotation was measured on CT scans using the Aaro-Dahlborn method.

Results: For AIS, the mean preoperative Cobb's angle, pelvic obliquity, and apical rotation values were $57.3^{\circ}, 2.8^{\circ}$, and $20.4^{\circ}$, respectively, and postoperatively they were $16.8^{\circ}, 1.1^{\circ}$ and $14.7^{\circ}$, respectively, showing significant correction. For NMS, the mean preoperative Cobb's angle, pelvic obliquity, and apical rotation values were $75.6^{\circ}, 13.7^{\circ}$, and $42.9^{\circ}$, respectively, and postoperatively they were $27.1^{\circ}, 5.8^{\circ}$, and $34.1^{\circ}$, respectively, also showing significant correction. There were no significant differences between AIS and NMS patients Cobb's angle $\mathrm{p}=0.306$, pelvic obliquity $\mathrm{p}=0.887$ and apical derotation $\mathrm{p}=0.113^{\circ}$. There were no differences in curve severity in the three groups (AIS, NMS $>80^{\circ}$ and NMS $\left\langle 80^{\circ}\right.$ ); or the correction of apical rotation ( $\mathrm{p}=0.25$ ), although less correction was achieved in the Cobb's angle in the $>80$ NMS group $(p=0.04)$.

Conclusions: Apical axial derotation can be achieved with posterior only pedicle screw fixation in NMS without anterior release, with comparable results in idiopathic scoliosis.

Key Words: Idiopathic scoliosis and neuromuscular scoliosis, Posterior only pedicle screw fixation, Computed tomography scan, Apical derotation

\section{Introduction}

Scoliosis is a three dimensional deformity ${ }^{1}$ with coronal angulations and axial rotation that need to be corrected simultaneously. Harrington rods produce poor control over rotational deformity ${ }^{2.3}$, and other instrumentation can better correct rotational deformity with coronal angulations. In scoliosis, the most profound rotation is observed in apical vertebrae ${ }^{4}$, although other vertebrae are also rotated, with treatment generally improving all vertebrae. Pedicle screws can best correct both deformities simultaneously and were chosen for this study.

Idiopathic scoliosis can be treated with different types of instrumentation for correcting vertebral rotation and the correction of Cobb's angle. In contrast, neuromuscular scoliosis (NMS), a rigid deformity, is not generally treated surgically to correct axial rotation. Here, we compared postoperative apical axial derotation in adolescent idiopathic and NMS treated with posterior pedicle screw fixation using

Received Jun 16, 2008; 1st revised Jul 27, 2008; 2nd revised Aug 25, 2008; 3rd revised Oct 20, 2008; accepted Oct 20, 2008

Corresponding author: Seung-Woo Suh, MD

Department of Orthopedic Surgery, Korea University Guro Hospital

\# 80 Guro-dong, Guro-gu, Seoul, 152-703, Korea

Tel: +82-2-865-1541, Fax: +82-2-867-1145, E-mail: spine@korea.ac.kr 
CT scans.

\section{Material and Methods}

Twelve patients with adolescent idiopathic scoliosis (AIS) and 16 patients with NMS were chosen for our study. All patients received surgery at our hospital in 2005 or 2006 with posterior only pedicle screw fixation followed by correction and fusion.

For AIS group (group A), the mean patient age ( 9 females and 3 males) at the time of operation was 14.1 years (Table 1). Nine patients had a major thoracic curve while 3 had major thoracolumbar curves. We excluded the patients who had double curves to maintain uniformity of the study. The average preoperative Cobb's angle was $57.3^{\circ}$, with flexibility of $38 \%$.

For the NMS group, the mean age of all the patients (5 cerebral palsy $[\mathrm{CP}], 6$ Duchenne muscular dystrophy [DMD] and 5 spinal muscular atrophy [SMA]) was 16.5 years. Only patients with a single curve were considered for this study to reduce errors, because derotation of double curves improves one rotation angle and worsens the other. Out of 16 patients included in this group, there were 11 thoracolumbar curves, 3 lumbar curves, and 2 thoracic curves. We have divided neuromuscular patients into two groups according to curve severity; group B (curve $<80^{\circ}, 8$ patients) with average Cobb angle of 55.5 (and group $\mathrm{C}$ (curve $>80^{\circ}, 8$ patients) with an average Cobb angle of $95.7^{\circ}$ (Table 2). Results were analyzed by idiopathic and NMS, as well as curve severity. Preoperatively, all the patients received a radiogram, CT scan, and pulmonary function test per our standard protocol.
All patients received surgery from a single spine surgeon with posterior only pedicle screw fixation, followed by correction and fusion with or without rib hump excision depending upon post-fixation appearance. During the operation, after full exposure using the standard posterior approach, pedicle screws were inserted bilaterally with freehand technique at all the levels and facet joints were thoroughly destroyed, including the apical and the adjacent levels to facilitate maximum rotational correction. Pre-contoured rods were then inserted over the pedicle screws bilaterally, followed by a standard derotation maneuver ${ }^{5,6}$ with or without in situ contouring of rods on both sides simultaneously. The rods were fixed by tightening the screw caps. Decortications of posterior laminae and posterior fusion were accomplished with bone grafts mixed with allografts. Multiple-layer wound closure was then performed and two drainage tubes inserted. All the patients underwent radiograms and CT scans, which were stored in our computerized PACS system with preoperative data, once they were hemodynamically stable and their drains were removed.

We calculated the coronal angulations by Cobb's angle ${ }^{7}$ and pelvic obliquity along a horizontal line on radiograms, while apical vertebral rotation was calculated on a CT scan with the Aaro-Dahlborn method ${ }^{4.8}$ from the mid-sagittal plane (Fig. 1). A well-trained, experienced spine fellow, familiar with all the techniques, performed all the calculations. We analyzed the preoperative and immediate postoperative Cobb's angle and apical axial derotation as average correction and percentages of correction according to curve severity and disease groups (group A, B, and C) using analysis of variance (ANOVA) tests.

The correction in Cobb's angle, pelvic obliquity, and apical axial derotation were compared with a paired t-test for
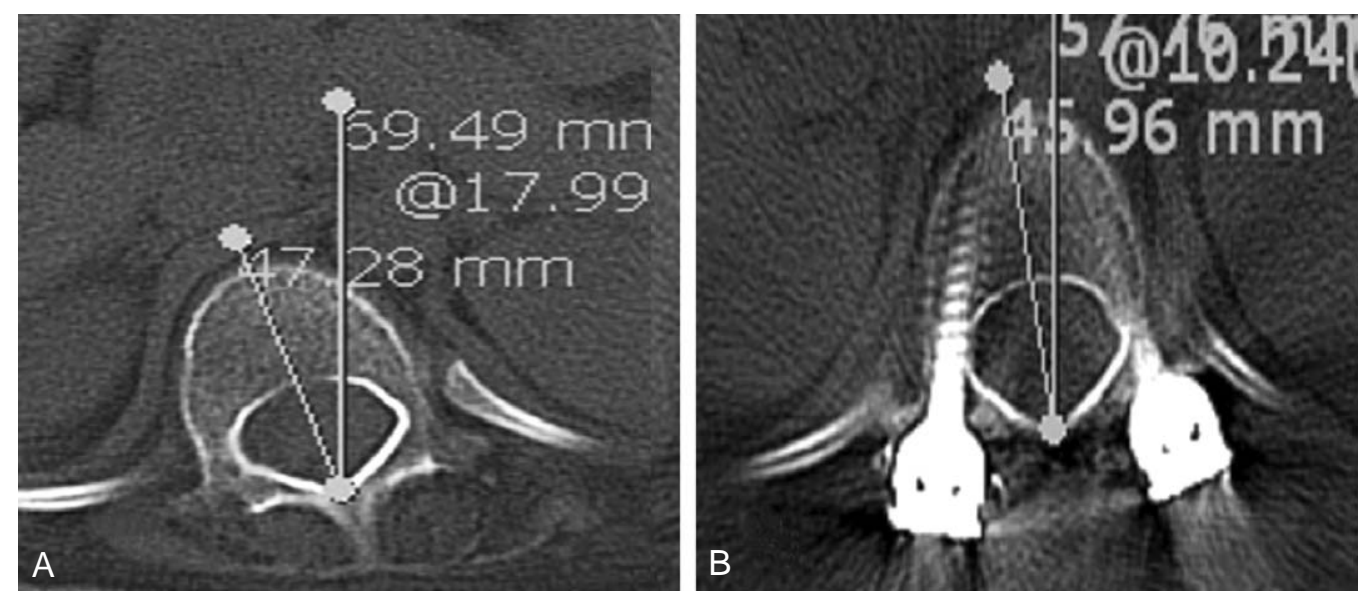

Fig. 1. Measurement of axial rotation in the mid-sagittal plane CT scan (A) preoperative and (B) postoperative. 
all groups. A p value of less than 0.05 was considered significant.

\section{Results}

The average follow-up was 26 months, ranging from 15 to 35 months. For the idiopathic scoliosis group, the average preoperative Cobb's angle, pelvic obliquity, and apical axial rotation were $57.3^{\circ}$ (range, 46 to $80^{\circ}$ ), $2.8^{\circ}$ (range, 0 to $8^{\circ}$ ) and $20.4^{\circ}$ (range, 9 to $36^{\circ}$ ), respectively. Postopera- tively, the average Cobb's angle, pelvic obliquity, and apical axial rotation were $16.8^{\circ}, 1.1^{\circ}$, and $14.7^{\circ}$ respectively. After surgery, the average correction was $71.4 \%$ ( $\mathrm{p}<0.0001$, paired t-test) for postoperative Cobb's angle, $51.1 \%$ $(\mathrm{p}=0.006$, paired $\mathrm{t}$-test) for pelvic obliquity, and $31.3 \%$ $(\mathrm{p}=0.002$, paired $\mathrm{t}$-test) for apical axial rotation (Table 1).

Similarly for the NMS group, the average preoperative Cobb's angle, pelvic obliquity, and apical axial rotation were $75.6^{\circ}$ (range, 40 to $112^{\circ}$ ), $13.7^{\circ}$ (range, 1 to $27^{\circ}$ ) and $42.9^{\circ}$ (range, 15 to $72^{\circ}$ ), respectively. Postoperatively, the average Cobb's angle, pelvic obliquity, and apical axial

Table 1. Pre and post operative Cobb's angle, pelvic obliquity and apical axial rotation for AIS group

\begin{tabular}{|c|c|c|c|c|c|c|c|c|c|c|}
\hline \multirow[b]{2}{*}{ No } & \multirow[b]{2}{*}{ Age } & \multirow[b]{2}{*}{ Sex } & \multirow[b]{2}{*}{ Diagnosis } & \multicolumn{3}{|c|}{ Cobb's angle } & \multicolumn{2}{|c|}{ Pelvic obliquity } & \multicolumn{2}{|c|}{ Apical Axial Rotation } \\
\hline & & & & Pre op & Level & Post op & Pre op & Post op & Pre op & Post op \\
\hline 1 & 13 & $\mathrm{~F}$ & AIS & 46 & $\mathrm{~T}$ & 17 & 4 & 3 & 21 & 15 \\
\hline 2 & 15 & $\mathrm{~F}$ & AIS & 80 & $\mathrm{~T}$ & 37 & 2 & 1 & 20 & 11 \\
\hline 3 & 13 & $\mathrm{M}$ & AIS & 48 & $\mathrm{~T}$ & 14 & 3 & 2 & 18 & 13 \\
\hline 4 & 14 & $\mathrm{~F}$ & AIS & 73 & $\mathrm{~T}$ & 16 & 3 & 2 & 20 & 19 \\
\hline 5 & 16 & $\mathrm{~F}$ & AIS & 54 & $\mathrm{~T}$ & 14 & 2 & 0 & 22 & 7 \\
\hline 6 & 14 & M & AIS & 50 & $\mathrm{~T}$ & 15 & 1 & 1 & 9 & 3 \\
\hline 7 & 13 & $\mathrm{~F}$ & AIS & 40 & $\mathrm{~T}$ & 16 & 8 & 2 & 18 & 20 \\
\hline 8 & 16 & F & AIS & 42 & TL & 4 & 1 & 0 & 18 & 6 \\
\hline 9 & 17 & F & AIS & 78 & $\mathrm{~T}$ & 37 & 2 & 1 & 36 & 30 \\
\hline 10 & 13 & $\mathbf{M}$ & AIS & 70 & $\mathrm{TL}$ & 8 & 5 & 1 & 29 & 29 \\
\hline 11 & 13 & $\mathrm{~F}$ & AIS & 50 & $\mathrm{~T}$ & 9 & 3 & 1 & 11 & 7 \\
\hline 12 & 13 & F & AIS & 57 & TL & 15 & 0 & 0 & 23 & 17 \\
\hline
\end{tabular}

AIS: adolescent idiopathic scoliosis, T: thoracic apex, TL: thoracolumbar apex.

Table 2. Pre and post operative Cobb's angle, pelvic obliquity and apical axial rotation for NMS group

\begin{tabular}{rrrrrrrrrrr}
\hline & & & \multicolumn{3}{c}{ Cobb's angle } & \multicolumn{2}{c}{ Pelvic obliquity } & \multicolumn{2}{c}{ Apical Axial Rotation } \\
No & Age & Sex & Diagnosis & Pre op & Level & Post op & Pre op & Post op & Pre op & Post op \\
\hline 1 & 16 & F & CP & 64 & TL & 27 & 15 & 13 & 40 & 30 \\
2 & 22 & M & CP & 40 & L & 17 & 8 & 4 & 34 & 20 \\
3 & 19 & M & CP & 52 & TL & 6 & 6 & 2 & 32 & 39 \\
4 & 23 & M & CP & 65 & T & 8 & 11 & 6 & 15 & 15 \\
5 & 21 & M & CP & 108 & TL & 39 & 15 & 5 & 60 & 50 \\
6 & 14 & M & DMD & 100 & TL & 38 & 17 & 5 & 58 & 46 \\
7 & 17 & M & DMD & 81 & TL & 59 & 22 & 7 & 72 & 57 \\
8 & 14 & M & DMD & 40 & L & 9 & 11 & 4 & 23 & 19 \\
9 & 12 & M & DMD & 66 & L & 28 & 26 & 2 & 41 & 29 \\
10 & 16 & M & DMD & 83 & TL & 36 & 16 & 2 & 48 & 44 \\
11 & 10 & M & DMD & 46 & TL & 13 & 10 & 8 & 20 & 17 \\
12 & 9 & F & SMA & 71 & T & 5 & 4 & 4 & 21 \\
13 & 28 & F & SMA & 92 & TL & 55 & 27 & 12 & 54 \\
14 & 13 & F & SMA & 112 & TL & 24 & 20 & 16 & 59 \\
15 & 18 & F & SMA & 108 & TL & 30 & 10 & 3 & 47 \\
16 & 13 & F & SMA & 82 & TL & 41 & 1 & 6 & 46 \\
\hline
\end{tabular}

NMS: neuromuscular, CP: cerebral palsy, DMD: duchene muscular dystrophy, SMA: spinal muscular atrophy, T: thoracic apex, TL: thoracolumbar apex, L: lumbar apex. 
rotation were $27.1^{\circ}, 5.8^{\circ}$, and $34.1^{\circ}$, respectively. After surgery, the average correction was $65.1 \%(\mathrm{p}<0.0001$, paired t-test) for Cobb's angle, $49.3 \%(\mathrm{p}=0.0008$, paired ttest) for pelvic obliquity, and $18.3 \%(\mathrm{p}=0.0003$, paired $\mathrm{t}$ test) for apical axial rotation (Table 2).

The correction rates were not different in the idiopathic and neuromuscular groups (Cobb's angle $\mathrm{p}=0.306$, pelvic obliquity $\mathrm{p}=0.887$ and apical derotation $\mathrm{p}=0.113$; unpaired t-test), despite less correction in apical rotation overall in NMS. Clinically, all patients exhibited postoperative improvement in walking ability, cosmetic appearance, and/or sitting balance, which improved quality of life.

The correction in Cobb's angle was significantly different in the three groups ( $\mathrm{p}=0.04$, ANOVA test) (Fig. 2) while correction in pelvic obliquity ( $\mathrm{p}=0.79$, ANOVA test) (Fig. 3) and apical derotation ( $\mathrm{p}=0.25$, ANOVA test) (Fig. 4) were not. Additionally we also compared the effect of correction in three diseases of NMS group: CP, DMD and SMA. The three different disease types, CP, DMD, and SMA, did not show differences in Cobb's angle ( $\mathrm{p}=0.54)$, pelvic obliquity $(\mathrm{p}=0.10)$ or apical derotation $(\mathrm{p}=0.46)$ by ANOVA.

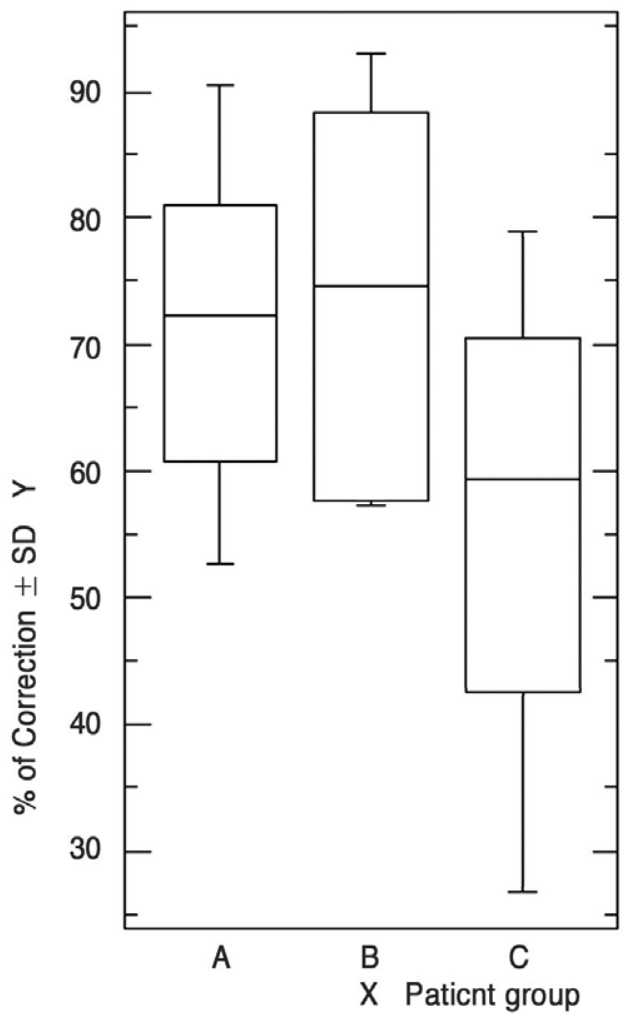

Fig. 2. Graph for analysis for Cobb's angle. The X-axis denotes scoliosis groups: A: AIS group, B: NMS $<80^{\circ}$, and C; NMS $>80^{\circ}$. The $\mathrm{Y}$-axis denotes $\%$ of correction in Cobb's angle with SD.

\section{Discussion}

Although, the apical vertebra shows maximum rotation', the upper and lower end vertebrae also exhibit rotational components in scoliosis. The vertebral and inter-vertebral axial rotation ${ }^{10}$ indicates the severity and rigidity of the scoliosis curve. Surgical success depends on the correction of the Cobb's angle, rotational angle, and translation of the vertebrae. Apical rotation outcomes are typically investigated in idiopathic scoliosis ${ }^{11,12}$ in the literature, with no reported outcomes in the neuromuscular group, probably because of the relatively small number of cases.

Here we have compared apical axial derotation in neuromuscular and AIS groups. Although different methods can measure the axial rotation of vertebrae $e^{12-15}$, computerized tomography is the most accurate. Since the pioneering work of Aaro-Dahlborn ${ }^{4.8}$ in 1980 to measure axial rotation on CT scans, several other methods have been developed to measure axial rotation. Gocen et al. ${ }^{16}$ in 1999 used a new technique to measure axial rotation by CT scan. Krismer et al. ${ }^{10}$

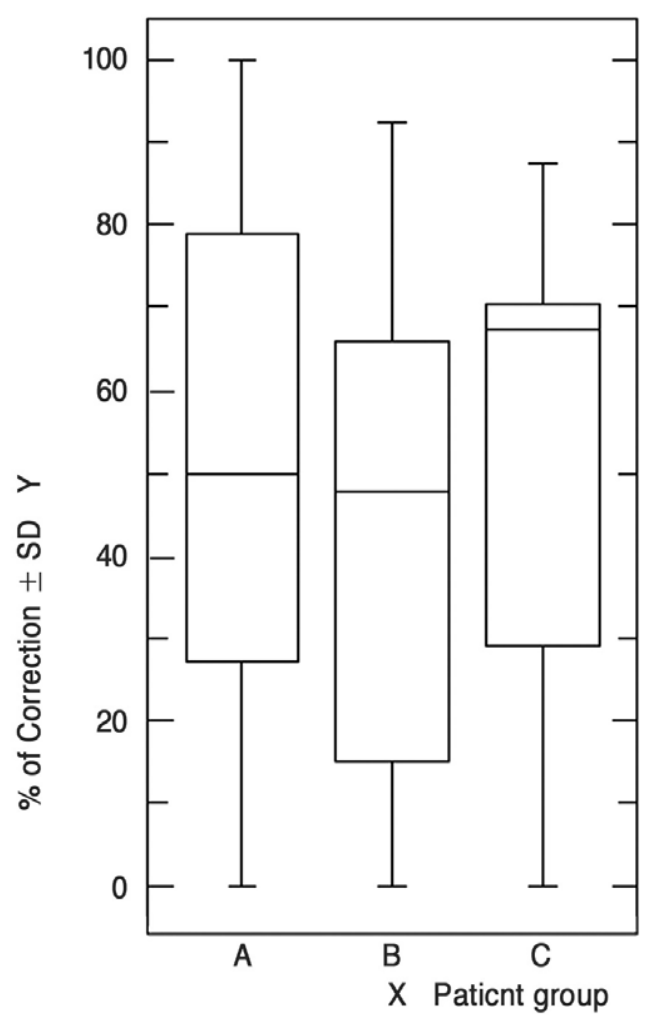

Fig. 3. Graph for analysis for pelvic obliquity. The $\mathrm{X}$-axis denotes scoliosis groups: A: AIS group, B: NMS $<80^{\circ}$, and C; NMS $>80^{\circ}$. The $\mathrm{Y}$-axis denotes $\%$ of correction in pelvic obliquity with SD. 


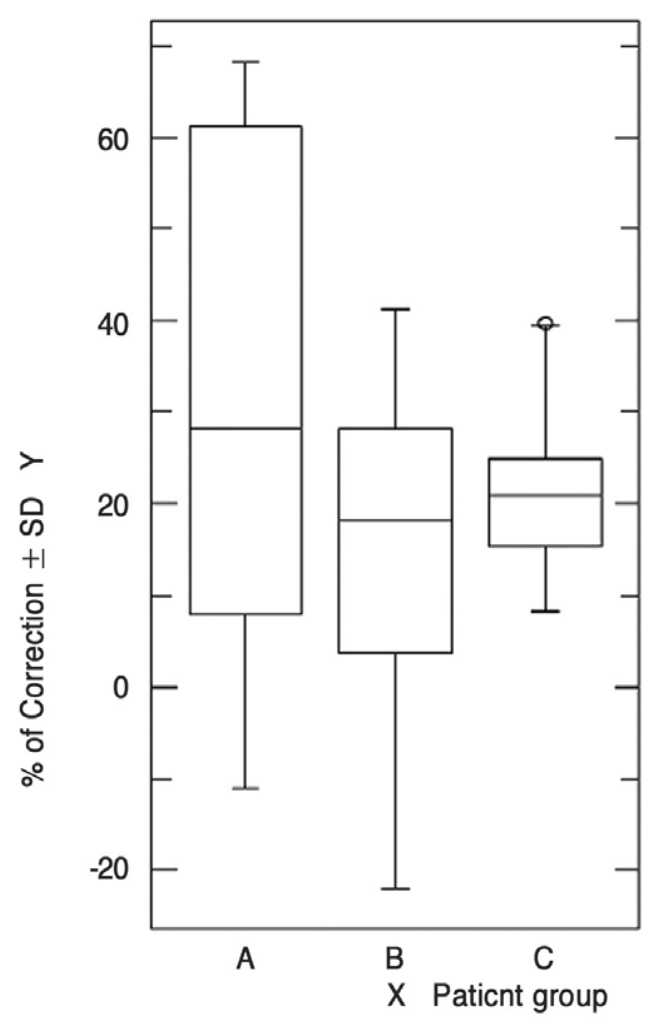

Fig. 4. Graph for analysis for apical derotation. The $\mathrm{X}$-axis denotes scoliosis groups: A: AIS group, B: NMS $<80^{\circ}$, and C; NMS $>80^{\circ}$. The Y-axis denotes $\%$ of correction in apical rotations with SD. AIS: idiopathic scoliosis, NMS: neuromuscular scoliosis.

studied 11 cadavers and found that the Aaro-Dahlborn method was superior to other techniques. We used the same method here.

Moreover, surgical correction with modern techniques is three-dimensional ${ }^{17}$. Aaro-Dahlborn ${ }^{3}$ evaluated 33 patients treated with Harrington instrumentation and found no axial derotation. Marchesi et al. ${ }^{18}$ used CT scans to measure derotation in four idiopathic scoliosis patients treated with Harrington instrumentation, plus seven with Luque instrumentation, and found an average derotation of $16 \%$ and $12 \%$, respectively, in the apical vertebrae. Using the same instrumentation, Ecker et al. ${ }^{19}$ found only $14 \%$ corrections with increase in rotation in some vertebrae. Bipedicular instrumentation can achieve maximum correction in Cobb's angle as well as axial rotation. In 1996, Jarvis and Greene ${ }^{20}$ studied Wisconsin segmental spinal instrumentation, a hybrid system with Harrington distraction rods, Luque rods, and button-wire constructs, in 24 idiopathic scoliosis patients and found $23 \%$ derotation in 22 curves and $12 \%$ deteriora- tion in seven curves. They included double-curve patterns, which we excluded. Cundy et al..$^{21}$ used the Aaro-Dahlborn method to study the effect of Cotrel-Dubosset instrumentation on rotation in 34 idiopathic scoliosis patients and reported $24 \%$ derotation in relation to the mid-sagittal plane. Suk et al. ${ }^{22}$ in 1995 first proposed the use of thoracic pedicle screws as a fixation option for treatment of AIS. Lonstein et al. ${ }^{23}$ in 1999, while studying coronal and sagittal plane correction in AIS using pedicle screw constructs or hybrid thoracic hook lumbar constructs noted a trend towards better correction of the main thoracic curve with pedicle screws, as was subsequently seen for lumbar curves as well ${ }^{24,25}$. Here we found a $71.4 \%$ correction in the coronal plane and a $31.3 \%$ derotation in the axial plane for AIS and a $65.1 \%$ correction in the coronal plane and a $18.3 \%$ derotation in the axial plane for NMS, which were not different $(p=0.30$ for Cobb's angle and $p=0.11$ for apical derotation; unpaired t-test).

No published data exists on apical derotation in NMS Schufflebarger et al. ${ }^{26}$ used CT scan to measure rotation in relation to the mid sagittal plane in 18 patients with NMS using Cotrel-Dubosset instrumentation with fixation up to the pelvis. His findings reveal an average correction in the frontal plane of $36^{\circ}$, a $42 \%$ correction, but did not measure axial rotation. Steib et al. ${ }^{24}$ studied derotation by in situ contouring of rods with pedicle screws in thoracic and lumbar curves in 10 idiopathic and 10 degenerative scoliosis patients and noted derotation ranging from $8^{\circ}$ to $10^{\circ}$ (62\% to $67 \%$ ). Although we used in situ contouring in a few cases, our main purpose was to prevent screw loosening from the pedicle. In 2003 Aubin et al. ${ }^{27}$ studied biomechanical modeling of posterior instrumentation of the scoliotic spine with Cotrel-Dubosset instrumentation using a three step procedure, and noted $18^{\circ}$ derotation in apical vertebrae, reflecting the kinematics of the rod-implant-vertebrae joint. Our all-pedicle screw construct with a posterior only approach produced comparable results with a similar derotation maneuver. In 2003, Basobas et al. ${ }^{28}$ demonstrated excellent results for selective anterior fusion for the treatment of NMS in 20 patients (most with meningomyelocele) in their retrospective study, but they did not comment on rotation. In 2002, Rhee et al..$^{29}$ did not find a difference in their sagittal plane comparison of AIS after anterior versus posterior instrumentation in 110 patients. Laohachroensombat et al. ${ }^{30}$ found a significant difference in apical derotation $(45 \%)$ in three dimensions after inserting pedicular screw plate constructs in 25 idiopathic scoliosis patients. 
We achieved nearly the same derotation in the CP, DMD, and SMA groups ( $\mathrm{p}=0.46$, ANOVA test), although we had small sample sizes and the results may depend on scoliosis severity. However, we found a $31 \%$ correction in apical rotation in the AIS group, but only an $18 \%$ change in the neuromuscular group.

\section{Conclusions}

In our retrospective study, we attained comparable changes in apical axial derotation in both groups, as well as in comparison of the AIS group with two severities of NMS. Although correction in the coronal plane was different among the groups A, B and C ( $\mathrm{p}=0.04$, ANOVA test), we noted similar derotations in the apical vertebrae. There is no other apical derotation data for NMS in the literature. However, the pedicle screw construct produces satisfactory outcomes in idiopathic scoliosis, prompting interest in future comparison studies of these two types of scoliosis.

\section{REFERENCES}

1. Kojima T, Kurokawa T: Quantitation of three-dimensional deformity of idiopathic scoliosis. Spine 1992; 17: S2229.

2. Benson DR, DeWald RL, Schultz AB: Harrington rod distraction instrumentation: its effect on vertebral rotation and thoracic compensation. Clin Orthop Relat Res 1977; 125: 40-44.

3. Aaro S, Dahlborn M: The effect of Harrington instrumentation on the longitudinal axis rotation of the apical vertebra and on the spinal and rib-cage deformity in idiopathic scoliosis studied by computer tomography. Spine 1982; 7 : 456-462.

4. Aaro S, Dahlborn M: Estimation of vertebral rotation and the spinal and rib cage deformity in scoliosis by computer tomography. Spine 1981; 6: 460-467.

5. Steib JP, Dumas R, Mitton D, Skalli W: Surgical correction of scoliosis by in situ contouring: a detorsion analysis. Spine 2004; 29: 193-199.

6. Chang KW: Cantilever bending technique for treatment of large and rigid scoliosis. Spine 2003; 28: 2452-2458.

7. Cobb JR: Outline for the study of scoliosis. American Academy of Orthopaedic Surgeons Instr Course Lect 1948; 5: 261-275.
8. Aaro S, Dahlborn M, Svensson L: Estimation of vertebral rotation in structural scoliosis by computer tomography. Acta Radiol Diagn 1978; 19: 990-992.

9. Deacon P, Flood BM, Dickson RA: Idiopathic scoliosis in three dimensions. A radiographic and morphometric analysis. J Bone Joint Surg Br 1984; 66: 509-512.

10. Krismer M, Sterzinger W, Haid C, Frischhut B, Bauer R: Axial rotation measurement of scoliotic vertebrae by means of computed tomography scans. Spine 1996; 21: 576-581.

11. Willers U, Transfeldt EE, Hedlund R: The segmental effect of Cotrel-Dubousset instrumentation on vertebral rotation, rib hump and the thoracic cage in idiopathic scoliosis. Eur Spine J 1996; 5: 387-393.

12. Perdriolle R, Vidal J: Morphology of scoliosis: threedimensional evolution. Orthopedics. 1987; 10: 909-915.

13. Nash CL Jr, Moe JH: A study of vertebral rotation. J Bone Joint Surg Am 1969; 51: 223-229

14. Suzuki S, Yamamuro T, Shikata J, Shimizu K, Iida H: Ultrasound measurement of vertebral in idiopathic scoliosis. J Bone Joint Surg Br 1989; 71: 252-255.

15. Bunnell WP: Vertebral rotation: simple method of measurement on routine radiographs. Orthop Trans 1985; 9: 114.

16. Gocen S, Havitcioglu H, Alici E: A new method to measure vertebral rotation from CT scans. Eur Spine J. 1999; 8: 261-265.

17. Ha JH, Lee JH, Ahn YJ, et al: The Explanation of Postoperative Change of Vertebral Rotation and Rib Hump Using 3 Dimensional Finite Element Scoliosis Model. J Korean Soc Spine Surg 2003; 10: 14-24.

18. Marchesi DG, Transfeldt EE, Bradford DS, Heithoff KB: Changes in vertebral rotation after Harrington and Luque instrumentation for idiopathic scoliosis. Spine 1992; 17: 775-780

19. Ecker ML, Betz RR, Trent PS, et al: Computer tomography evaluation of Cotrel-Dubousset instrumentation in idiopathic scoliosis. Spine 1988; 13: 1141-1144.

20. Jarvis JG, Greene RN: Adolescent idiopathic scoliosis. Correction of vertebral rotation with use of Wisconsin segmental spinal instrumentation. J Bone Joint Surg Am 1996; 78: $1707-1712$

21. Cundy PJ, Paterson DC, Hillier TM, Sutherland AD, Stephen JP, Foster BK: Cotrel-Dubousset instrumentation and vertebral rotation in adolescent idiopathic scoliosis. $\mathrm{J}$ Bone Joint Surg Br 1990; 72: 670-674.

22. Suk SI, Lee CK, Kim WJ, Chung YJ, Park YB: Seg- 
mental pedicle screw fixation in the treatment of thoracic idiopathic scoliosis. Spine 1995; 20: 1399-1405.

23. Lowenstein JE, Matsumoto H, Vitale MG, et al: Coronal and sagittal plane correction in adolescent idiopathic scoliosis: a comparison between all pedicle screw versus hybrid thoracic hook lumbar screw constructs. Spine 2007; 32: 448-452.

24. Steib JP, Ducrocq X, Averous C, Bogorin J: Surgical correction of lumbar scoliosis: a comparison of different techniques. Results. Eur J Orthop Surg \& Traumatol 1999; 9: 151-156.

25. Boos N, Webb JK: Pedicle screw fixation in spinal disorders: a European view. Eur Spine J 1997; 6: 2-18.

26. Neustadt JB, Shufflebarger HL, Cammisa FP: Spinal fusions to the pelvis augmented by Cotrel-Dubousset instrumentation for neuromuscular scoliosis. J Pediatr
Orthop 1992; 12: 465-469.

27. Aubin CE, Petit Y, Stokes IA, Poulin F, Gardner-Morse M, Labelle H: Biomechanical modeling of posterior instrumentation of the scoliotic spine. Comput Methods Biomech Biomed Engin 2003; 6: 27-32.

28. Basobas L, Mardjetko S, Hammerberg K, Lubicky J: Selective anterior fusion and instrumentation for the treatment of neuromuscular scoliosis. Spine 2003; 28: S245248.

29. Rhee JM, Bridwell KH, Won DS, Lenke LG, Chotigavanichaya C, Hanson DS: Sagittal plane analysis of adolescent idiopathic scoliosis: the effect of anterior versus posterior instrumentation. Spine. 2002; 27: 2350-2356.

30. Laohacharoensombat $\mathbf{W}$, Jaovisidha $\mathbf{S}$, Wajanavisit $\mathbf{W}$, Suppaphol S: Apical derotation in the treatment of idiopathic scoliosis. J Med Assoc Thai 2005; 88: S58-64. 